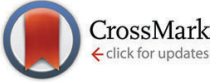

Cite this: Phys. Chem. Chem. Phys., 2016, 18, 4842

Received 20th November 2015 Accepted 18th January 2016

DOI: $10.1039 / c 5 c p 07092 a$

www.rsc.org/pccp

\title{
Oligothiophene wires: impact of torsional conformation on the electronic structure $\dagger$
}

\author{
D. A. Kislitsyn, ${ }^{a}$ B. N. Taber, ${ }^{a}$ C. F. Gervasi, ${ }^{a}$ L. Zhang, ${ }^{\text {b }}$ S. C. B. Mannsfeld, ${ }^{c}$ \\ J. S. Prell, ${ }^{a}$ A. L. Briseno ${ }^{b}$ and G. V. Nazin*a
}

\begin{abstract}
Charge transport in polymer- and oligomer-based semiconductor materials depends strongly on the structural ordering of the constituent molecules. Variations in molecular conformations influence the electronic structures of polymers and oligomers, and thus impact their charge-transport properties. In this study, we used Scanning Tunneling Microscopy and Spectroscopy (STM/STS) to investigate the electronic structures of different alkyl-substituted oligothiophenes displaying varied torsional conformations on the $\mathrm{Au}(111)$ surface. STM imaging showed that on $\mathrm{Au}(111)$, oligothiophenes self-assemble into chain-like structures, binding to each other via interdigitated alkyl ligands. The molecules adopted distinct planar conformations with alkyl ligands forming cis- or trans- mutual orientations. For each molecule, by using STS mapping, we identify a progression of particle-in-a-box-like states corresponding to the LUMO, LUMO+1 and LUMO+2 orbitals. Analysis of STS data revealed very similar unoccupied molecular orbital energies for different possible molecular conformations. By using density functional theory calculations, we show that the lack of variation in molecular orbital energies among the different oligothiophene conformers implies that the effect of the Au-oligothiophene interaction on molecular orbital energies is nearly identical for all studied torsional conformations. Our results suggest that cis-trans torsional disorder may not be a significant source of electronic disorder and charge carrier trapping in organic semiconductor devices based on oligothiophenes.
\end{abstract}

\section{Introduction}

Solution-processable thiophene-based polymers and oligomers represent an important class of organic semiconductor materials ${ }^{1,2}$ with potential applications in field-effect transistors, ${ }^{3-5}$ lightemitting diodes, ${ }^{6,7}$ photodetectors ${ }^{8}$ and photovoltaic devices. ${ }^{9-11}$ The electronic structures of polymers and oligomers, and consequently their charge transport properties, are determined, to a significant degree, by the conformations of their $\pi$-conjugated backbones. $^{12-14}$ Molecular conformations not only govern the extent of electronic conjugation, but are also interdependent with molecular packing, ${ }^{15,16}$ which controls intermolecular charge transport in polymer- and oligomer-based thin films. ${ }^{17}$ While bulk molecular packing is of central importance in defining the electronic transport characteristics, ${ }^{18}$ the latter are also often

\footnotetext{
${ }^{a}$ Department of Chemistry and Biochemistry, Materials Science Institute, Oregon Center for Optical, Molecular and Quantum Science, University of Oregon, 1253 University of Oregon Eugene, OR 97403, USA. E-mail: gnazin@uoregon.edu ${ }^{b}$ Department of Polymer Science and Engineering, University of MassachusettsAmherst, Silvio O. Conte National Center for Polymer Research, 120 Governors Drive Amherst, MA 01003, USA

${ }^{c}$ Center for Advancing Electronics Dresden, Dresden University of Technology, 01062 Dresden, Germany

$\dagger$ Electronic supplementary information (ESI) available. See DOI: 10.1039/c5cp07092a
}

strongly affected by the properties of molecular interfaces with other materials used in electronic devices, where molecular structures can be quite different from those of the bulk. ${ }^{19,20}$ Examples of such interfaces include the molecule-dielectric interfaces, found in field-induced conduction channels in transistors, ${ }^{21-23}$ and molecule-electrode interfaces. ${ }^{24-26}$ Furthermore, the thin film morphology can be strongly impacted by the structure of the molecular layer at the interface. ${ }^{27}$ The nature of the interface has a profound impact on the molecular morphology and self-organization, which are defined by the competition between the intermolecular and molecule-surface interactions. For this reason, molecular structures at interfaces with solids and other molecular layers have been a subject of numerous studies, with techniques based on scanning tunneling microscopy (STM) being particularly powerful in the absence of long-range order due to their ability to resolve local molecular structures. Many STM studies have focused on molecular self-assembly in the regime of weak molecule-surface interactions simulating molecule-dielectric interfaces, with molecules often deposited on highly-oriented pyrolytic graphite surfaces. ${ }^{28-34}$ The resulting structures are typically dominated by the intermolecular interactions between the ligands, attached to thiophene backbones in order to enhance solubility and facilitate processing.

Several STM studies have addressed the regime of stronger molecule-surface interactions with molecules deposited on 
metal surfaces simulating molecule-electrode interfaces. ${ }^{30,35-42}$ A common observation of these studies was the existence of conformational polymorphisms of both polythiophenes ${ }^{40,41}$ and oligothiophenes, ${ }^{36-39}$ with the overall degree of disorder being higher than that found for more weakly interacting surfaces. ${ }^{30,40}$ Understanding the degree of electronic disorder associated with such conformational polymorphisms is important in view of the potentially significant impact of molecular conformation on the electronic structure. Theoretical and optical spectroscopic investigations of organic donor-acceptor molecules incorporating thiophene donors revealed that unoccupied frontier electronic levels were largely unaffected by cis-trans conformational isomerization, finding variations in LUMO energies due to rotational disorder of less than $90 \mathrm{meV}^{43,44}$ STM offers the capability to probe the impact of conformational polymorphism on molecular electronic structure directly via scanning tunneling spectroscopy (STS), which has been used to investigate oligothiophene electronic structures on both strongly and weakly interacting surfaces. ${ }^{29,30,45-49}$ However, the role of conformational effects in defining the molecular electronic structure has so far only been investigated in very short oligothiophenes (incorporating only four thiophene rings), where few electronic states are accessible to STS due to their relatively high energies. ${ }^{50,51}$

Here we report a conformation-resolved STM/STS study of alkylsubstituted oligothiophenes adsorbed on the $\mathrm{Au}(111)$ surface. To obtain a more complete physical picture of the oligothiophene properties on $\mathrm{Au}(111)$, we investigated two types of oligothiophenes with similar structures: molecules incorporating eight thiophene rings and four alkyl ligands (we will refer to these molecules as $8 \mathrm{~T}$ in the following), as well as molecules incorporating seven thiophene rings and three alkyl ligands (7T in the following). Both types of molecules are sufficiently long to be considered as finite-length models for probing conformational effects in application-relevant alkyl-substituted thiophene-based polymers and oligomers. The Au(111) surface serves as a model of Au metal electrodes often used in proof-of-principle organic semiconductor devices. We obtained STS maps of oligothiophene molecules to identify the nature of molecular orbitals, and found that all molecules displayed particle-in-a-box-like progressions of electronic states. Further, we found that different oligothiophene conformations, despite considerable structural differences, result in nearly indistinguishable molecular electronic structures. We use density functional theory (DFT) calculations to show that the electronic structures of oligothiophenes of different torsional conformations (in the gas phase) are quite similar. The lack of conformational sensitivity in our experimental electronic structure data thus suggests that the Au-oligothiophene interaction is relatively insensitive to the specific molecular torsional conformation.

\section{Experimental methods}

STM and STS measurements were performed at $\sim 20 \mathrm{~K}$ using a home-built ultra-high vacuum (UHV) cryogenic (closed-cycle cryostat-based) STM system incorporating a STM scanner from
RHK Technology. ${ }^{52}$ Atomically clean $\mathrm{Au}(111)$ on mica was prepared in UHV by multiple cycles of Ne-ion bombardment followed by $\sim 300{ }^{\circ} \mathrm{C}$ anneals. 8T and $7 \mathrm{~T}$ molecules were prepared by Briseno et al. ${ }^{53}$ The composition was verified with nanoelectrospray ionization mass spectrometry (Fig. S1, ESI $\dagger$ ). Submonolayers of oligothiophene molecules were deposited on the Au surface via in situ sublimation with the sample held at room temperature and vacuum pressure not exceeding $10^{-10}$ Torr. STS spectra were measured in constant-height mode using the lock-in technique, with a modulation frequency of $570 \mathrm{~Hz}$ and a bias modulation of $50 \mathrm{mV}$. This allowed for the direct measurement of the local differential conductance $(\mathrm{d} I / \mathrm{d} V)$ of the sample, which serves as a measure to the local density of electronic states. ${ }^{54}$ DFT computations were performed with Gaussian $09^{55}$ using B3LYP/6-31G* ${ }^{* 6,57}$ and analyzed with Multiwfn. ${ }^{58}$

\section{Results and discussion}

\section{Molecular adsorption configurations from STM imaging}

After deposition on the Au(111) surface, STM imaging revealed that $8 \mathrm{~T}$ and $7 \mathrm{~T}$ molecules self-assembled into chain-like aggregates comprised of varying numbers of molecules (Fig. 1a, Fig. S2a and S3a, ESI $\dagger$ ). High-resolution STM images show that the molecules bind to each other with their alkyl ligands, forming interdigitating patterns (Fig. 1b and Fig. S2b, ESI $\dagger$ ).

Adsorption of oligothiophenes was highly correlated with the structural features of the $\mathrm{Au}(111)$ surface associated with the $\mathrm{Au}(111) 22 \times \sqrt{3}$ surface reconstruction, which results in the formation of regions with fcc (face-centered cubic) and hcp (hexagonal close-packed) surface structures separated by reconstruction "ridges" visible in STM images (dashed lines in Fig. 1c, Fig. S2c and S3b, ESI $\dagger$ ). The molecular aggregates primarily formed in the fcc regions of the $\mathrm{Au}(111)$ surface, and were almost entirely absent from the hcp regions (Fig. 1c, Fig. S2c and S3b, ESI $\dagger$ ), consistent with results for shorter oligothiophenes. ${ }^{45,50}$ This preferential adsorption has been attributed to different reactivities of the fcc and hcp regions, a result of varied coordination of the surface $\mathrm{Au}$ atoms in the two regions. ${ }^{50}$ STM images of $8 \mathrm{~T}$ and $7 \mathrm{~T}$ backbones also showed molecular profiles of $\sim 2 \AA$ with typical variations of $<0.1 \AA$, consistent with flat molecular backbones. ${ }^{49}$

The molecular aggregates showed reproducible registry with the $\mathrm{Au}(111)$ surface lattice, as can be observed by comparing molecular orientations with the ridge-like surface reconstruction features (Fig. 1c for 8T, Fig. S2c and S3b for 7T, ESI $\dagger$ ). In particular, in the straight sections of fcc regions, where the $\mathrm{Au}$ crystal structure is more regular, the oligothiophene backbones were preferentially oriented along the $\langle 110\rangle$ directions of $\mathrm{Au}(111)$, orthogonal to the growth-directions of molecular aggregates corresponding approximately to the $\langle 112\rangle$ directions (Fig. 1c, Fig. S2c and S3b, ESI $\dagger$ ). This backbone orientation is analogous to that observed for shorter four-thiophene oligomers (4T in the following) on $\mathrm{Au}(111),{ }^{50}$ which is a direct consequence of the similarities in intermolecular and molecule-surface interactions for both systems. In particular, the preferred orientations 

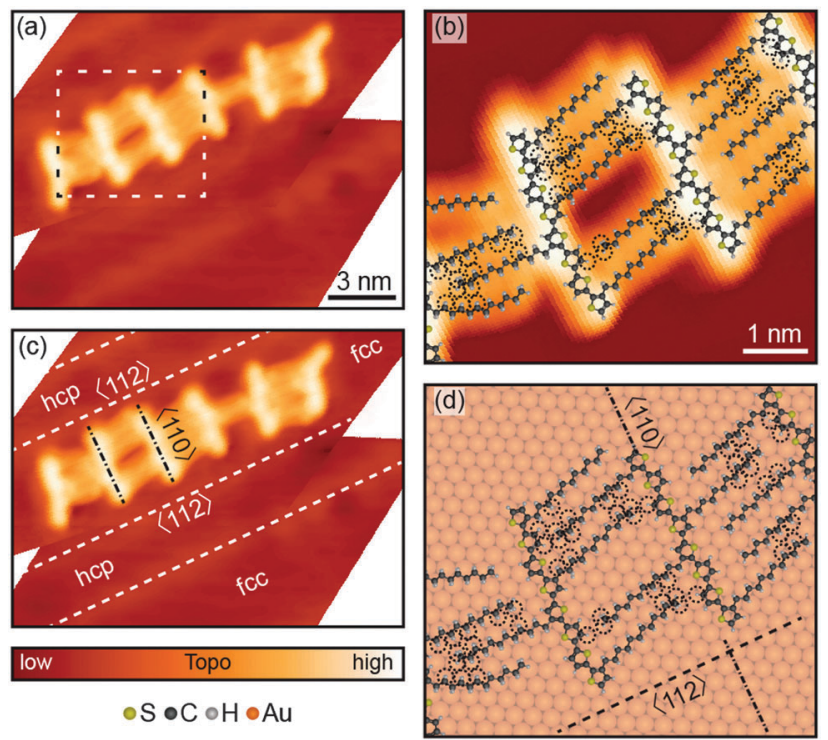

Fig. 1 Adsorption of $8 \mathrm{~T}$ molecules on $\mathrm{Au}(111)$. (a) STM image [set point $100 \mathrm{mV}, 5 \mathrm{pA}$ ] of an aggregate of oligothiophene molecules absorbed on the Au(111) surface. (b) Close-up STM topography of the region confined by the dotted rectangle in (a). Atomic models of $8 \mathrm{~T}$ molecules are overlaid on the STM image. The atomic models show that molecules are attached to each other via alkyl substituents. The thiophene rings comprising the DDQT backbones are nearly flat on the Au(111) surface, as determined by STM topographies. (c) STM image from (a) with indicated molecular orientations and $\mathrm{Au}(111)$ crystallographic directions and highlighted surface-reconstruction ridges. The oligothiophene backbones of $8 \mathrm{~T}$ molecules are aligned along the $\langle 110\rangle$ directions of the $A u(111)$ surface, perpendicular to the straight sections of surface-reconstruction ridges which run parallel to the $\langle 112\rangle$ directions. (d) Model of $8 \mathrm{~T}$ molecules from (b) matched to the Au(111) surface lattice. Au(111) crystallographic directions are indicated. Dashed circles indicate the van der Waals radii of the hydrogen atoms.

of $4 \mathrm{~T}$ molecules along the $\langle 110\rangle$ directions have been attributed to the charge-transfer interaction involving $\mathrm{S}$ atoms of the thiophene units comprising $4 \mathrm{~T}$ molecules and the Au surface. For individual thiophene units, this interaction results in preferential adsorption on Au top sites, ${ }^{59}$ with a local energy minimum of $40 \mathrm{meV} .^{60}$ The tendency of $4 \mathrm{~T}$ oligothiophenes to align along the $\langle 110\rangle$ directions on $\mathrm{Au}(111)$ has been explained by the fact that this orientation leads to the best matching of all four S-atoms of $4 \mathrm{~T}$ to the top sites of the $\mathrm{Au}(111)$ surface lattice. ${ }^{50}$ In accordance with this picture, locations of S-atoms in $8 \mathrm{~T}$ and $7 \mathrm{~T}$ molecules show similar patterns (Fig. 1d and Fig. S2d, ESI $\dagger$ ), even though some deviations from perfect topsite placement are evident. The existence of these deviations is not unexpected, since the longer molecular structures of $7 \mathrm{~T}$ and $8 \mathrm{~T}$ molecules are more difficult to match to the $\mathrm{Au}(111)$ lattice. For example, while in $4 \mathrm{~T}$ molecules the distance between the S-atoms of the outer thiophene rings $(1.19 \mathrm{~nm})$ was closely matched to four Au atoms along the $\langle 110\rangle$ direction $(1.15 \mathrm{~nm})$, for $8 \mathrm{~T}$ molecules the distance between the S-atoms of the outer thiophene rings $(\sim 2.8 \mathrm{~nm})$ is a poorer match to $\mathrm{Au}(111)$ surface lattice sites, with the best match corresponding to ten lattice constants $(2.89 \mathrm{~nm})$. The lack of commensurability between the molecular structures and the $\mathrm{Au}(111)$ surface may also be the reason for the commonly observed deviations from linearity of $7 \mathrm{~T}$ and $8 \mathrm{~T}$ backbones, in contrast to $4 \mathrm{~T}$ molecules, which appeared straight in all STM images. ${ }^{50}$

Another important factor that likely impacted the shapes of $7 \mathrm{~T}$ and $8 \mathrm{~T}$ backbones is the intermolecular interactions originating from the alkyl ligands. These include both alkylalkyl interactions as well as interactions involving terminal methyl groups of alkyl ligands and thiophene units of neighboring molecules. For example, the strength of the alkyl-alkyl interaction can be expected to be substantial given that for a pair of dodecane molecules the interaction energy is estimated to be $0.4-0.5 \mathrm{eV}{ }^{61}$ This interaction tends to affect individual molecules differently due to the widely varied inter-digitation patterns of alkyl substituents connecting neighboring molecules (Fig. 1b and Fig. S2b, ESI $\dagger$ ), and may contribute to the observed deviations from linearity for the oligothiophene backbones. Indeed, this argument is supported by the fact that despite the variations in the oligothiophene backbone shapes, the patterns formed by alkyl chains show highly reproducible angles, especially when close alkyl-alkyl contacts are formed (Fig. 1b and Fig. S2b, ESI $\dagger$ ). The observed orientations of tightly packed alkyl ligands on the $\mathrm{Au}(111)$ lattice form angles of $\sim 12^{\circ}$ with respect to the $\langle 110\rangle$ direction (Fig. 1d and Fig. S2d, ESI $\dagger$ ). This ligand orientation is intermediate between those of the gas-phase molecules (ligand nearly orthogonal to the backbones), and alkanes self-assembled on $\mathrm{Au}(111)$, which typically align themselves approximately along the $\langle 110\rangle$ directions $^{62}$ (with theoretical calculations giving an angle of $\sim 7^{\circ}$ ). ${ }^{63}$ This observation suggests that orientations of the alkyl ligands are also affected by the local bonding orientation and placement of the oligothiophene backbones.

The specific interdigitating structures formed by alkyl ligands of neighboring molecules depend on the particular torsional conformations adopted by the corresponding thiophene backbones. Oligothiophene aggregates studied in our work contained a variety of molecules adopting different torsional conformations (corresponding to the different possible mutual orientations of thiophene units along the oligothiophene backbone, as can be seen in Fig. 1b and Fig. S2b, ESI $\dagger$ ), which is one of the reasons for varied intermolecular binding interactions. In addition to controlling the structure of oligothiophene aggregates, torsional conformations can be expected to control the electronic structures of the corresponding molecules, since changing the mutual orientation of individual thiophene units can affect the energies of molecular orbitals. From the point of view of electronic applications based on oligothiophene materials, it is then important to understand the extent of electronic structure variations caused by the existence of different torsional conformers, as well as the physical driving forces responsible for the diversity of torsional conformations in oligothiophene thin films.

The observed tendency of $8 \mathrm{~T}$ molecules to form relatively ordered aggregates suggests that after deposition, these molecules have sufficient energy (at room temperature) to be able to freely migrate on the surface on the $\mathrm{Au}(111)$ surface. Moreover, the well-ordered assembly of interconnecting alkyl ligands suggests that molecular torsional transformations readily occur 
in these conditions. This is illustrated, for example, by the fact that alkyl ligands unattached to neighbouring molecules were almost never observed in STM imaging, with one particular manifestation of this being the fact that molecules located at the ends of the oligothiophene aggregates typically assumed a conformation with all alkyl ligands facing the rest of the aggregate, whenever possible (a rare exception to this is the left molecule in Fig. S2a (ESI $\dagger$ ), where one of the ligands is facing away from the molecular aggregate due to interference from a ligand of a neighbouring molecule).

\section{Scanning tunneling spectroscopy}

We investigated the effect of length and conformation on oligothiophene electronic structure by using scanning tunneling spectroscopy (STS) to measure the local electronic structure of $8 \mathrm{~T}$ and $7 \mathrm{~T}$ molecules adsorbed on the $\mathrm{Au}(111)$ surface. Specifically, these measurements were carried out by recording the differential conductance (derivative of the tunneling current, $\mathrm{d} I / \mathrm{d} V$ ), which is representative of the local electronic density of states (DOS). By recording the differential conductance as a function of the applied bias voltage (which serves as the energy scale), we obtain energy-dependent DOS spectra (see Experimental methods for further details). ${ }^{54}$ In total, 55 straight oligothiophene molecules were studied using this approach (see Fig. S4, ESI $\dagger$ ), and STS maps were obtained for 31 molecules, as described below.
Among the different possible conformations of $8 \mathrm{~T}$ molecules, we focus on three representative conformations: CCC (orientation of ligands corresponding to a cis-cis-cis combination, Fig. 2a), TTT (trans-trans-trans ligand orientations, Fig. 3a), and CTT (cis-trans-trans ligand orientations, Fig. 4a). While we are unable to discern the orientations of thiophenes lacking alkyl chains, it is likely that, since the oligothiophene backbones are straight, neighboring thiophenes are preferentially oriented $\sim 180^{\circ}$ relative to each other, as oligothiophene backbones containing neighboring thiophenes oriented $\sim 0^{\circ}$ relative to each other have a tendency to bend due to the steric hindrance between neighboring thiophenes. ${ }^{30}$ By recording progressions of STS spectra along the oligothiophene backbones (corresponding spatial paths shown in Fig. 2b, 3b and $4 \mathrm{~b}$ ) we obtain DOS maps showing the spatial landscape of molecular electronic states (Fig. 2c, 3c and 4c). Here we focus on the unoccupied states because the occupied molecular orbitals were not clearly distinguishable in the DOS spectra due to the structured background originating from the d-bands of the $\mathrm{Au}(111)$ surface. All three DOS patterns (Fig. 2c, 3c and 4c) show a very high degree of similarity. Each map contains features attributable to a progression of three orbitals-LUMO, LUMO+1 and LUMO+2-showing the characteristic particle-in-a-box spatial distributions: the LUMO+1 and LUMO+2 orbitals show one and two spatial nodes respectively, while the LUMOs are relatively featureless along the molecular backbones (Fig. 2d, 3d and 4d).
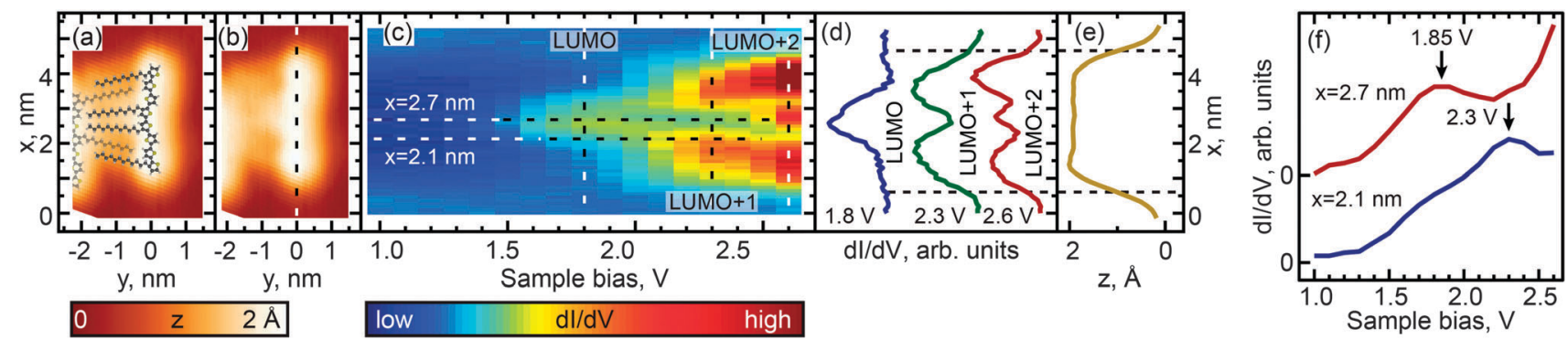

Fig. 2 Spatial (STS) DOS mapping across a CCC conformer of 8T molecules. (a) STM image with an overlaid atomic model of the CCC-8T molecule. (b) STM image from (a) showing the path of mapping (dashed line). (c) DOS as a function of the bias voltage and position $x$ along the path shown in (b). (d) LUMO, LUMO+1 and LUMO+2 DOS along the path shown in (b), obtained at voltages corresponding to the vertical dashed lines in (c). These voltages were chosen to maximize the contributions of the corresponding individual orbitals. Curves are shifted and normalized for clarity. (e) Backbone profile ( $z$ height vs. $x$ coordinate) along the dashed line from (b). (f) Individual STS spectra from (c) measured at $x=2.1$ and $2.7 \mathrm{~nm}$ as indicated by horizontal lines in (c). Spectra are shifted for clarity. The LUMO state manifests itself as a peak at $1.85 \mathrm{~V}$ in the spectrum measured at $x=2.7 \mathrm{~nm}$, while LUMO+1 is observed as a peak at $2.3 \mathrm{~V}$ in the spectrum measured at $x=2.1 \mathrm{~nm}$. Only a shoulder of the LUMO+2 states is observed at $2.6 \mathrm{~V}(x=2.7 \mathrm{~nm})$.
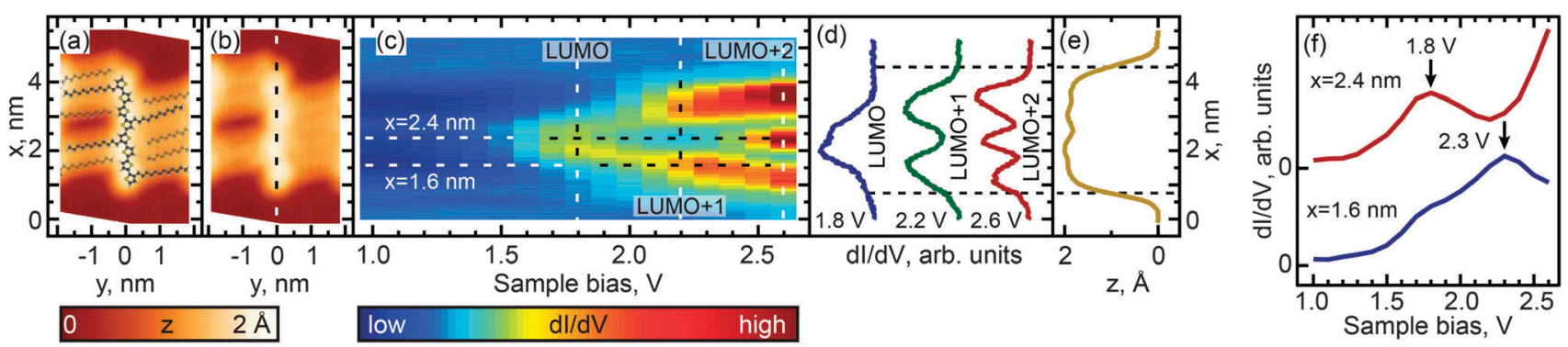

Fig. 3 Same as Fig. 2 for a TTT conformer of 8 T molecules. The molecule is situated in the center of the molecular aggregate, with alkyl chains on both sides (see Fig. S1d, ESI†). 

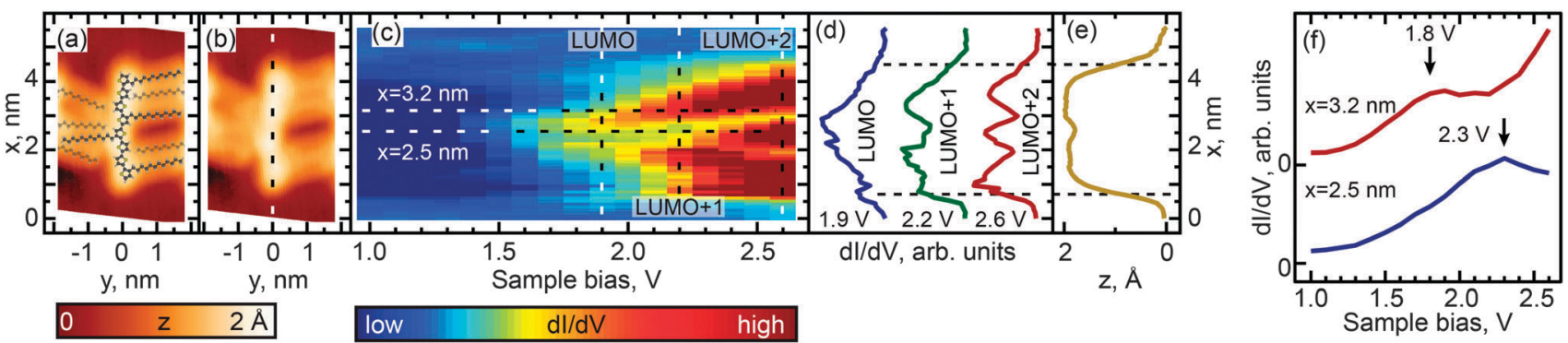

Fig. 4 Same as Fig. 2 for a CTT conformer of $8 \mathrm{~T}$ molecules.
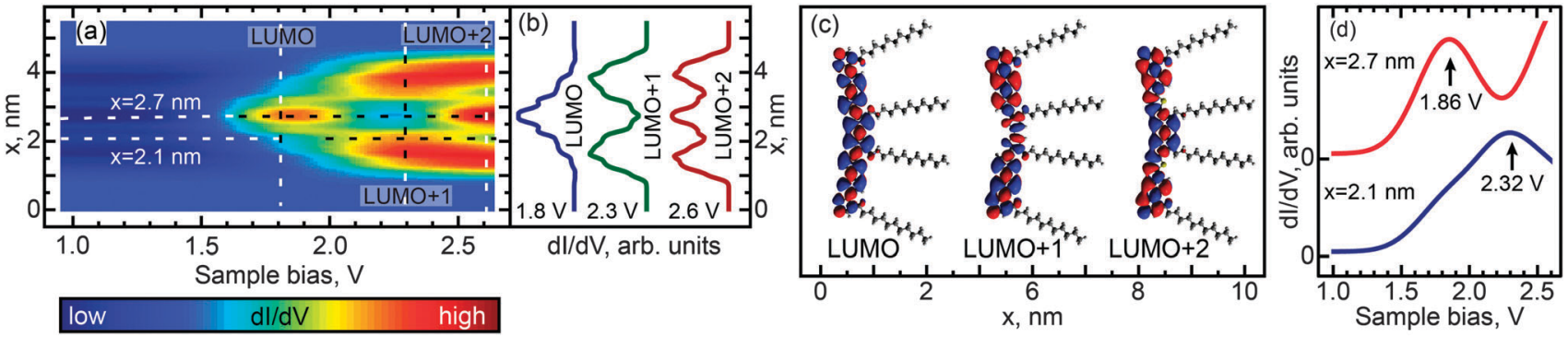

Fig. 5 Calculated electronic DOS for a CCC conformer of 8T molecules. (a) DOS (compare to Fig. 2c) as a function of the bias voltage and position $x$ along the path similar to that shown in Fig. 2b. (b) LUMO, LUMO+1 and LUMO+2 DOS (compare to Fig. 2d) along the same path as in (a), obtained at voltages corresponding to the vertical dashed lines in (a). These voltages were chosen to maximize the contributions of the corresponding individual orbitals. Curves are shifted and normalized for clarity. (c) Three-dimensional representations of LUMO, LUMO+1 and LUMO+2 showing nodal patterns (along the molecular backbone) characteristic of the particle-in-a-box nature of these states (no nodes for LUMO, one node for LUMO+1 and two nodes for LUMO+2). (d) Individual DOS spectra from (a) measured at spatial locations indicated by horizontal lines in (a). Spectra are shifted for clarity. The LUMO state manifests itself as a peak at $1.86 \mathrm{~V}$ (top curve), while LUMO+1 is observed as a peak at $2.32 \mathrm{~V}$ (bottom curve). Electronic structure calculations were performed with density functional theory (DFT) calculations using B3LYP/6-31G*

The assignment of LUMO, LUMO+1 and LUMO+2 orbitals in Fig. 2-4 is supported by DFT calculations carried out for $8 \mathrm{~T}$ molecules in gas phase (Fig. 5-7). Indeed, for CCC, TTT and CTT conformations, DOS patterns similar to those of Fig. 2c, 3c and $4 \mathrm{c}$ are found in calculations (Fig. 5a, 6a and 7a). For each molecular conformation, the patterns are formed by three states, LUMO, LUMO+1 and LUMO+2 with particle-in-a-box-like spatial structures (Fig. 5b, 6b and 7b) similar to those in Fig. 2d, 3d and $4 \mathrm{~d}$. The particle-in-a-box-nature of these states is evident from the corresponding calculated wavefunctions showing the characteristic nodal patterns (Fig. 5c, 6c and 7c).

The LUMO+1 and LUMO+2 orbitals appear more delocalized relative to the LUMO orbitals (compare DOS profiles in Fig. 2d, $3 \mathrm{~d}$ and $4 \mathrm{~d}$ to topography profiles in Fig. $2 \mathrm{e}$, 3e and $4 \mathrm{e}$ ), which is a consequence of several factors. First, due to the longer spatial wavelength of the lowest-energy particle-in-a-box state, the probability density of this state changes on a longer scale and therefore is suppressed over a longer spatial range at the ends of the oligothiophene backbone. In addition, higher-energy states are generally more spatially extended due to the lower effective tunneling barrier. Finally, the fact that the tunneling current (identical for all measured spectra) is composed of contributions from each unoccupied state means that the LUMO contribution is further suppressed via topographic effects when the tip is positioned near the ends of the oligothiophene backbone, where higher-energy states have larger contributions to the tunneling current (the feedback loop was opened at a bias of $2.6 \mathrm{~V}$ for all STS maps, so that the tip height was therefore
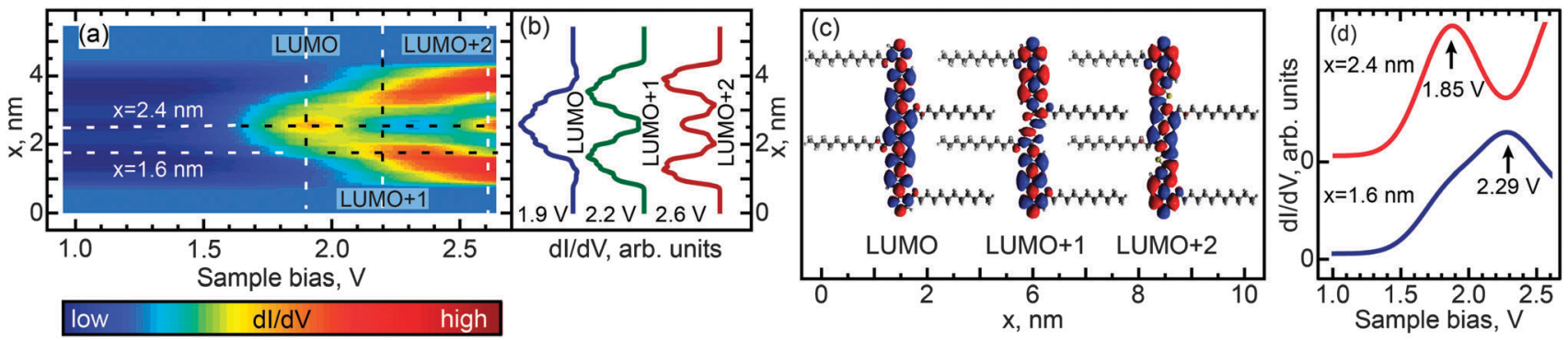

Fig. 6 Same as Fig. 5 for a TTT conformer of $8 \mathrm{~T}$ molecules. 

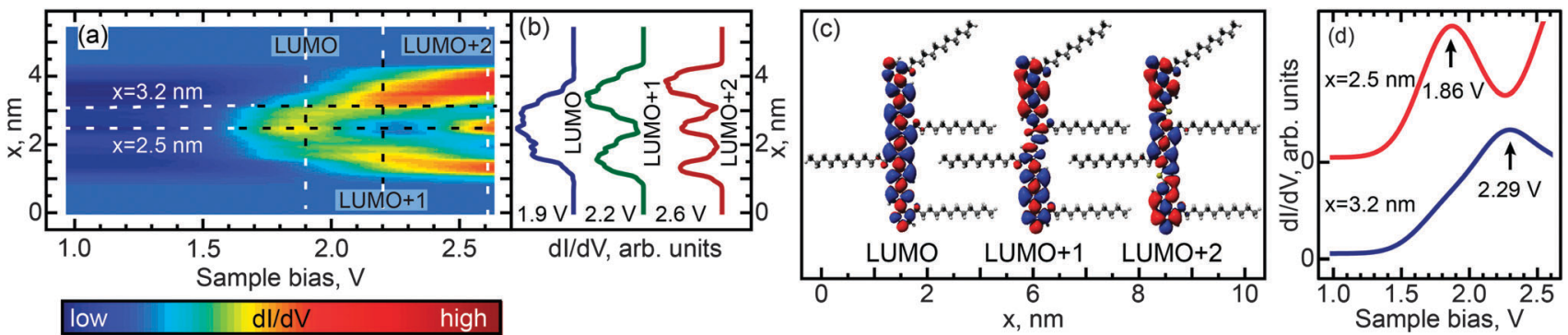

Fig. 7 Same as Fig. 6 for a CTT conformer of 8T molecules.

determined by contributions from the first three unoccupied molecular orbitals). A similar relationship between the localization scales of the LUMO, LUMO+1 and LUMO+2 orbitals is reproduced in our theoretical calculations (Fig. 5b, 6b and 7b).

While the unoccupied molecular orbital energies are the same within experimental error for all conformations, there are asymmetries observed in DOS intensity across the molecular backbones (Fig. 2d, 3d and 4d). DFT calculations for the CCC and TTT conformers do not exhibit asymmetry in DOS intensity (Fig. 5b and 6b). It is likely that the observed asymmetric experimental DOS intensity (Fig. 2d and 3d) is due to factors other than conformational variation for these conformers. DFT results for the CCT conformer, however, show uneven LUMO, LUMO+1 and LUMO+2 intensity across the molecular backbone (Fig. 7b), suggesting that asymmetric torsional conformation variations can lead to asymmetry in DOS intensity.

Despite the completely different torsional conformations of the molecules in Fig. 2-4, their orbital energies are essentially the same (1.8 V for LUMO and $2.3 \mathrm{~V}$ for LUMO+1, as determined from Fig. 2f, 3f, and 4f) within the experimental error $(\sim 50 \mathrm{mV})$. These observations are mirrored by the calculated state energies (obtained from Fig. 5d, 6d and 7d), which are very similar for all torsional conformations, as summarized in Fig. 8. Because our calculations were carried out for molecules in the gas phase, the similarity of the experimentally determined and calculated orbital energies suggests that the oligothiophene interaction with the $\mathrm{Au}(111)$ surface is relatively insensitive to the molecular conformation, with any differences between conformers being less than $50 \mathrm{mV}$. To further test this conclusion, we studied 7T molecules with analogous conformations to those of 8T (Fig. S5-S7, ESI $\dagger$ ). Because of their different structure and reduced symmetry, the interaction of $7 \mathrm{~T}$ molecules with $\mathrm{Au}(111)$ may be different from that of $8 \mathrm{~T}$ molecules, which could potentially lead to a different magnitude of electronic structure variations for different $7 \mathrm{~T}$ conformers. Similarly to $8 \mathrm{~T}$ molecules, we identify three representative conformations: CC (orientation of ligands corresponding to a cis-cis combination, Fig. S5a, ESI $\dagger$ ), TT (trans-trans ligand orientations, Fig. S6a, ESI $\dagger$ ), and CT (cis-trans ligand orientations following an unsubstituted section of the backbone, Fig. S7a, ESI $\dagger$ ). Analogously to the case of $8 \mathrm{~T}$ molecules, DOS maps of different conformers of $7 \mathrm{~T}$ oligothiophenes (Fig. S5c, S6c and S7c, ESI $\dagger$ ) show patterns attributable to progressions of three particle-in-a-box-like orbitals, LUMO, LUMO+1 and LUMO+2, consistent with theoretical calculations

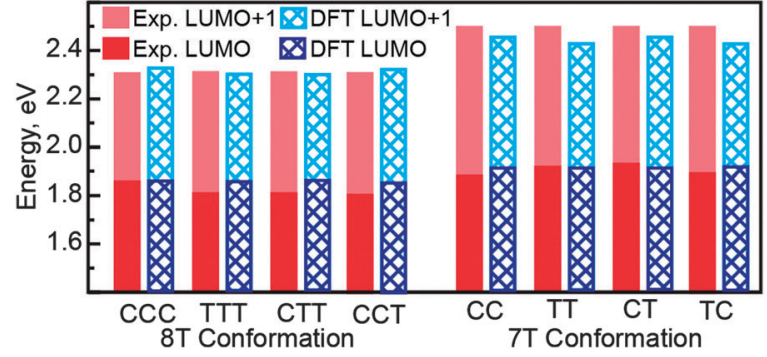

Fig. 8 Experimental (red solid bars) and theoretical (blue textured bars) LUMO and LUMO+1 energies for different torsional conformers of alkylsubstituted $8 \mathrm{~T}$ and $7 \mathrm{~T}$ oligothiophenes. Electronic orbitals for $7 \mathrm{~T}$ molecules are upshifted as compared to the $8 \mathrm{~T}$ molecules due to their shorter thiophene backbone lengths.

(Fig. S8-S10, ESI $\dagger$ ). As with the $8 \mathrm{~T}$ molecules, all studied conformers of the $7 \mathrm{~T}$ molecules showed, within the $50 \mathrm{mV}$ experimental error, nearly the same orbital energies $(1.9 \mathrm{~V}$ for LUMO and $2.5 \mathrm{~V}$ for LUMO+1), which are close to the theoretically predicted values summarized in Fig. 8.

Another example of the lack of sensitivity of the oligothiophene electronic structure to molecular conformation is provided by our recent work on $4 \mathrm{~T}$ molecules, where similar orbital energies were found for both cis and trans conformations, and the variation in molecular orbital energies were found to be attributable to the local variations in the $\mathrm{Au}(111)$ surface reactivity caused by the $22 \times \sqrt{3}$ surface reconstruction. ${ }^{50}$ In the present study, similar orbital energy variations with standard deviations of $40-60 \mathrm{mV}$ (depending on the molecular structure and conformation), comparable to the experimental error $(\sim 50 \mathrm{mV})$ were found. Thus, because the oligothiophene unoccupied molecular orbital energies were found to be insensitive to the torsional conformation of molecules with a wide range of structures, it is likely that conformational differences in the oligothiophene interaction with the $\mathrm{Au}(111)$ surface are on the scale of a few tens of meV.

\section{Conclusions}

Our results, obtained using STS spectroscopy, demonstrate that different torsional conformers of oligothiophene molecules with different lengths and (alkyl) substitutional patterns show molecular orbital energies nearly independent of the molecular conformations. While these results are consistent with 
theoretical simulations for oligothiophenes in the gas phase, the experimental demonstration of similar unoccupied molecular orbital energies (within $50 \mathrm{meV}$ ) across multiple planar molecular conformation, as demonstrated in our work, suggests that the oligothiophene interaction with the $\mathrm{Au}(111)$ surface on molecular energy levels in general is relatively insensitive to the molecular conformation. This is an important result for applications utilizing charge transport through thin films based on longer substituted oligothiophenes and polythiophenes, where significant local variations in conformational structure are often found. ${ }^{30,32,40,41}$ The insensitivity of the oligothiophene molecular electronic structure to torsional conformation presented here may mean a low degree of electronic disorder, and consequently, lower probability of charge carrier trapping within molecular backbones in polyand oligo-thiophene materials. Indeed, molecular orbital energy variations found in our STS measurements are comparable to the energetic disorder (20-40 meV) characteristic of high-mobility $\left(\sim 0.1 \mathrm{~cm}^{2} \mathrm{~V}^{-1} \mathrm{~s}^{-1}\right)$ polythiophene samples. ${ }^{64}$ Thus, while the molecular conformational and packing structures do affect the trap density in bulk poly- and oligo-thiophene materials, ${ }^{65}$ the present work suggests that in molecular films with predominating planar molecular conformations, the electronic disorder may be reduced.

\section{Acknowledgements}

The STM instrument used in this work was constructed with support from the National Science Foundation under Grant DMR-0960211. Acknowledgment is made to the Donors of the American Chemical Society Petroleum Research Fund for support of this research (Grant 52732-DNI6). B. N. T. and G. V. N. gratefully acknowledge support from the Center for Sustainable Materials Chemistry through the NSF CCI Grant CHE-1102637. L. Z. and A. L. B. gratefully acknowledge the Center for Hierarchical Manufacturing (CMMI-0531171). S. C. B. M. acknowledges support by the Deutsche Forschungsgemeinschaft (DFG) within the Cluster of Excellence "cfaed".

\section{References}

1 L. Zhang, N. S. Colella, B. P. Cherniawski, S. C. B. Mannsfeld and A. L. Briseno, ACS Appl. Mater. Interfaces, 2014, 6, 5327-5343.

2 L. Xu, L. Yang and S. Lei, Nanoscale, 2012, 4, 4399-4415.

3 I. McCulloch, M. Heeney, C. Bailey, K. Genevicius, I. MacDonald, M. Shkunov, D. Sparrowe, S. Tierney, R. Wagner, W. Zhang, M. L. Chabinyc, R. J. Kline, M. D. McGehee and M. F. Toney, Nat. Mater., 2006, 5, 328-333.

4 A. R. Murphy, J. M. J. Fréchet, P. Chang, J. Lee and V. Subramanian, J. Am. Chem. Soc., 2004, 126, 1596-1597.

5 C. Videlot-Ackermann, J. Ackermann, H. Brisset, K. Kawamura, N. Yoshimoto, P. Raynal, A. El Kassmi and F. Fages, J. Am. Chem. Soc., 2005, 127, 16346-16347.
6 M. Mazzeo, V. Vitale, F. Della Sala, M. Anni, G. Barbarella, L. Favaretto, G. Sotgiu, R. Cingolani and G. Gigli, Adv. Mater., 2005, 17, 34-39.

7 R. H. Friend, R. W. Gymer, A. B. Holmes, J. H. Burroughes, R. N. Marks, C. Taliani, D. D. C. Bradley, D. A. D. Santos, J. L. Bredas, M. Logdlund and W. R. Salaneck, Nature, 1999, 397, 121-128.

8 L. Tong, C. Li, F. E. Chen, H. Bai, L. Zhao and G. Q. Shi, J. Phys. Chem. C, 2009, 113, 7411-7415.

9 R. Fitzner, E. Mena-Osteritz, A. Mishra, G. Schulz, E. Reinold, M. Weil, C. Körner, H. Ziehlke, C. Elschner, K. Leo, M. Riede, M. Pfeiffer, C. Uhrich and P. Bäuerle, J. Am. Chem. Soc., 2012, 134, 11064-11067.

10 L. Zhang, F. Liu, Y. Diao, H. S. Marsh, N. S. Colella, A. Jayaraman, T. P. Russell, S. C. B. Mannsfeld and A. L. Briseno, J. Am. Chem. Soc., 2014, 136, 18120-18130.

11 K. Cnops, B. P. Rand, D. Cheyns, B. Verreet, M. A. Empl and P. Heremans, Nat. Commun., 2014, 5, 3406.

12 C. Schubert, J. T. Margraf, T. Clark and D. M. Guldi, Chem. Soc. Rev., 2015, 44, 988-998.

13 B. Q. Xu, X. L. Li, X. Y. Xiao, H. Sakaguchi and N. J. Tao, Nano Lett., 2005, 5, 1491-1495.

14 B. Capozzi, E. J. Dell, T. C. Berkelbach, D. R. Reichman, L. Venkataraman and L. M. Campos, J. Am. Chem. Soc., 2014, 136, 10486-10492.

15 N. Koch, J. Phys.: Condens. Matter, 2008, 20, 184008.

16 A. Höfer, K. Duncker, M. Kiel, S. Wedekind and W. Widdra, Phys. Rev. B: Condens. Matter Mater. Phys., 2011, 83, 075414.

17 H. Sirringhaus, P. J. Brown, R. H. Friend, M. M. Nielsen, K. Bechgaard, B. M. W. Langeveld-Voss, A. J. H. Spiering, R. A. J. Janssen, E. W. Meijer, P. Herwig and D. M. de Leeuw, Nature, 1999, 401, 685-688.

18 Y. Wu, P. Liu, B. S. Ong, T. Srikumar, N. Zhao, G. Botton and S. Zhu, Appl. Phys. Lett., 2005, 86, 142102.

19 S. Duhm, G. Heimel, I. Salzmann, H. Glowatzki, R. L. Johnson, A. Vollmer, J. P. Rabe and N. Koch, Nat. Mater., 2008, 7, 326-332.

20 R. Joseph Kline, M. D. McGehee and M. F. Toney, Nat. Mater., 2006, 5, 222-228.

21 A. Dodabalapur, L. Torsi and H. E. Katz, Science, 1995, 268, 270-271.

22 J. Huang, J. Sun and H. E. Katz, Adv. Mater., 2008, 20, 2567.

23 S. Fabiano, C. Musumeci, Z. Chen, A. Scandurra, H. Wang, Y.-L. Loo, A. Facchetti and B. Pignataro, Adv. Mater., 2012, 24, 951.

24 G. R. Dholakia, M. Meyyappan, A. Facchetti and T. J. Marks, Nano Lett., 2006, 6, 2447-2455.

25 J. E. Lyon, A. J. Cascio, M. M. Beerbom, R. Schlaf, Y. Zhu and S. A. Jenekhe, Appl. Phys. Lett., 2006, 88, 222109.

26 H. Ishii, K. Sugiyama, E. Ito and K. Seki, Adv. Mater., 1999, 11, 605 .

27 S. Wang, A. Kiersnowski, W. Pisula and K. Müllen, J. Am. Chem. Soc., 2012, 134, 4015-4018.

28 E. Mena-Osteritz, A. Meyer, B. M. W. Langeveld-Voss, R. A. J. Janssen, E. W. Meijer and P. Bäuerle, Angew. Chem., Int. Ed., 2000, 39, 2679-2684. 
29 Z.-Y. Yang, H.-M. Zhang, G.-B. Pan and L.-J. Wan, ACS Nano, 2008, 2, 743-749.

30 Z.-Y. Yang, H.-M. Zhang, C.-J. Yan, S.-S. Li, H.-J. Yan, W.-G. Song and L.-J. Wan, Proc. Natl. Acad. Sci. U. S. A., 2007, 104, 3707-3712.

31 E. Mena-Osteritz, M. Urdanpilleta, E. El-Hosseiny, B. Koslowski, P. Ziemann and P. Bauerle, Beilstein J. Nanotechnol., 2011, 2, 802-808.

32 X. J. Ma, Y. Guo, T. Wang and Z. H. Su, J. Chem. Phys., 2013, 139, 014701.

33 T. Jaroch, M. Knor, R. Nowakowski, M. Zagorska and A. Pron, Phys. Chem. Chem. Phys., 2008, 10, 6182-6189.

34 B. Grévin, P. Rannou, R. Payerne, A. Pron and J. P. Travers, J. Chem. Phys., 2003, 118, 7097-7102.

35 L. Wang, B. J. V. Tongol, S.-L. Yau, T. Otsubo and K. Itaya, Langmuir, 2010, 26, 7148-7152.

36 F. Nishiyama, K. Ogawa, S. Tanaka and T. Yokoyama, J. Phys. Chem. B, 2008, 112, 5272-5275.

37 K. Duncker, M. Kiel, A. Hoefer and W. Widdra, Phys. Rev. B: Condens. Matter Mater. Phys., 2008, 77, 155423.

38 T. Yokoyama, Y. Kogure, M. Kawasaki, S. Tanaka and K. Aoshima, J. Phys. Chem. C, 2013, 117, 18484-18487.

39 B. J. V. Tongol, L. Wang, S.-L. Yau, T. Otsubo and K. Itaya, Langmuir, 2010, 26, 982-989.

40 Y. F. Liu, K. Krug and Y. L. Lee, Nanoscale, 2013, 5, 7936-7941.

41 S. Förster, E. Kohl, M. Ivanov, J. Gross, W. Widdra and W. Janke, J. Chem. Phys., 2014, 141, 164701.

42 D. Wang, L.-J. Wan and C.-L. Bai, Mater. Sci. Eng., R, 2010, 70, 169-187.

43 L. V. Brownell, K. A. Robins, Y. Jeong, Y. Lee and D. C. Lee, J. Phys. Chem. C, 2013, 117, 25236-25247.

44 B. He, A. B. Pun, D. Zherebetskyy, Y. Liu, F. Liu, L. M. Klivansky, A. M. McGough, B. A. Zhang, K. Lo, T. P. Russell and L. W. Wang, J. Am. Chem. Soc., 2014, 136, 15093-15101.

45 B. Koslowski, A. Tschetschetkin, N. Maurer, E. Mena-Osteritz, P. Bauerle and P. Ziemann, Beilstein J. Nanotechnol., 2011, 2, 561-568.

46 G. Reecht, H. Bulou, F. Scheurer, V. Speisser, B. Carrière, F. Mathevet and G. Schull, Phys. Rev. Lett., 2013, 110, 056802.

47 J. Repp, P. Liljeroth and G. Meyer, Nat. Phys., 2010, 6, 975-979.

48 F. Jäckel, U. G. E. Perera, V. Iancu, K. F. Braun, N. Koch, J. P. Rabe and S. W. Hla, Phys. Rev. Lett., 2008, 100, 126102.

49 E. Varene, Y. Pennec and P. Tegeder, Chem. Phys. Lett., 2011, 515, 141-145.
50 B. N. Taber, D. A. Kislitsyn, C. F. Gervasi, S. C. B. Mannsfeld, L. Zhang, A. L. Briseno and G. V. Nazin, ACS Appl. Mater. Interfaces, 2015, 19732-19742.

51 D. A. Kislitsyn, B. N. Taber, C. F. Gervasi, S. C. B. Mannsfeld, L. Zhang, A. L. Briseno and G. V. Nazin, J. Phys. Chem. C, 2015, 26959-26967.

52 J. D. Hackley, D. A. Kislitsyn, D. K. Beaman, S. Ulrich and G. V. Nazin, Rev. Sci. Instrum., 2014, 85, 103704.

53 L. Zhang, N. S. Colella, F. Liu, S. Trahan, J. K. Baral, H. H. Winter, S. C. B. Mannsfeld and A. L. Briseno, J. Am. Chem. Soc., 2012, 135, 844-854.

54 C. J. Chen, Introduction to Scanning Tunneling Microscopy, Oxford University Press, New York, 2nd edn, 2008.

55 M. J. Frisch, G. W. Trucks, H. B. Schlegel, G. E. Scuseria, M. A. Robb, J. R. Cheeseman, G. Scalmani, V. Barone, B. Mennucci, G. A. Petersson, H. Nakatsuji, M. Caricato, X. Li, H. P. Hratchian, A. F. Izmaylov, J. Bloino, G. Zheng, J. L. Sonnenberg, M. Hada, M. Ehara, K. Toyota, R. Fukuda, J. Hasegawa, M. Ishida, T. Nakajima, Y. Honda, O. Kitao, H. Nakai, T. Vreven, J. A. Montgomery Jr., J. E. Peralta, F. Ogliaro, M. J. Bearpark, J. Heyd, E. N. Brothers, K. N. Kudin, V. N. Staroverov, R. Kobayashi, J. Normand, K. Raghavachari, A. P. Rendell, J. C. Burant, S. S. Iyengar, J. Tomasi, M. Cossi, N. Rega, N. J. Millam, M. Klene, J. E. Knox, J. B. Cross, V. Bakken, C. Adamo, J. Jaramillo, R. Gomperts, R. E. Stratmann, O. Yazyev, A. J. Austin, R. Cammi, C. Pomelli, J. W. Ochterski, R. L. Martin, K. Morokuma, V. G. Zakrzewski, G. A. Voth, P. Salvador, J. J. Dannenberg, S. Dapprich, A. D. Daniels, Ö. Farkas, J. B. Foresman, J. V. Ortiz, J. Cioslowski and D. J. Fox, Gaussian 09 Revision C.01, Gaussian, Inc., Wallingford CT, 2009.

56 A. D. Becke, J. Chem. Phys., 1993, 98, 5648.

57 P. J. Stephens, F. J. Devlin, C. F. Chabalowski and

M. J. Frisch, J. Phys. Chem., 1994, 98, 11623-11627.

58 T. Lu and F. Chen, J. Comput. Chem., 2012, 33, 580-592.

59 J. Zhou, Y. X. Yang, P. Liu, N. Camillone, III and M. G. White, J. Phys. Chem. C, 2010, 114, 13670-13677.

60 K. Tonigold and A. Gross, J. Chem. Phys., 2010, 132, 224701. 61 A. Goursot, T. Mineva, R. Kevorkyants and D. Talbi, J. Chem. Theory Comput., 2007, 3, 755-763.

62 H. M. Zhang, Z. X. Xie, B. W. Mao and X. Xu, Chem. - Eur. J., 2004, 10, 1415-1422.

63 R. J. Baxter, G. Teobaldi and F. Zerbetto, Langmuir, 2003, 19, 7335-7340.

64 H. Sirringhaus, Adv. Mater., 2005, 17, 2411-2425.

65 A. Salleo, T. W. Chen, A. R. Völkel, Y. Wu, P. Liu, B. S. Ong and R. A. Street, Phys. Rev. B: Condens. Matter Mater. Phys., 2004, 70, 115311. 